\title{
Estimating potential soil erosion for environmental services in a sugarcane growing area using multisource remote sensing data
}

Mulianga, B. ${ }^{1,2,3}$, Bégué A. ${ }^{1}$, Simoes M. ${ }^{1,4}$, Todoroff P. ${ }^{5}$ and Clouvel P. ${ }^{3}$

CIRAD UMR TETIS, Maison de la Télédétection, 500 rue J-F. Breton, Montpellier, F-34093 France

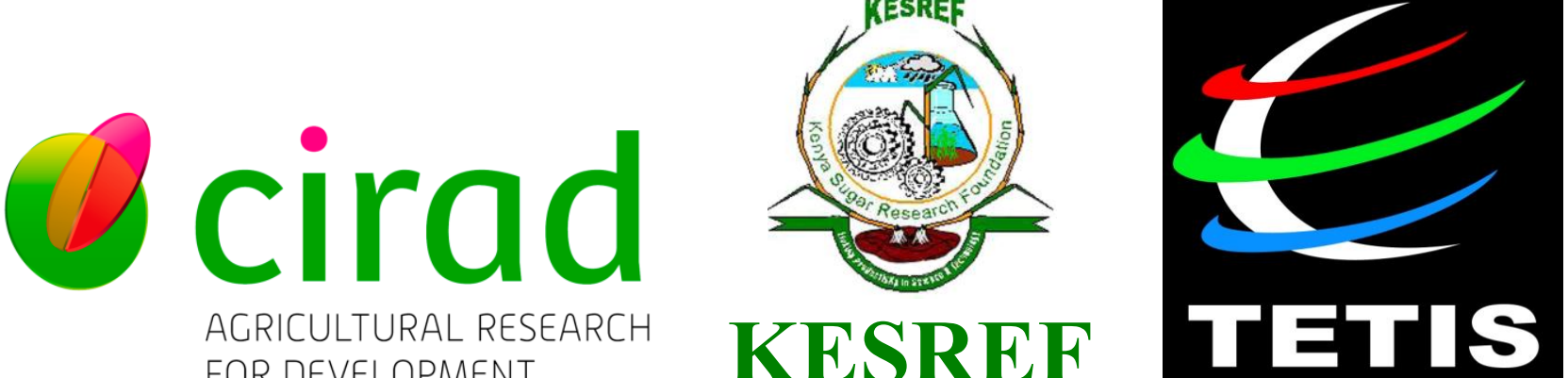

Embrapa

KESREF, Kisumu-Miwani Road, P.O Box 44 - 40100, Kisumu, Kenya.

KESREF

Contacts

4 EMBRAPA SOLOS and UERJ/PPGMA, Rua Jardim Botânico, 1024, Rio de Janeiro, Brazil,
${ }^{4}$ CIRAD UPR SCA, Avenue Agropolis, Montellier Cedex 5-34398 France

agnes.begue@cirad.fr

${ }^{5}$ CIRAD UPR SCA, Station de Ligne-Paradis, 7 chemin de l'IRAT, Saint-Pierre, Réunion, F-97410 France

bmulianga@gmail.com

\section{Introduction}

Estimation of potential soil erosion in the western region of Kenya, is crucial in ascertaining environmental services that are provided by main land uses for sustainable land management. The objective of this study was to investigate the soil erosion control service offered by sugarcane farming in Kisumu-Kibos sugar zone, using remote sensing data and an erosion model. The study focused on sensitivity of erosion risk to the vegetation conditions at landscape scale. Different type of remote sensing data is used to qualify the spatial and temporal variability of the agro environmental conditions.

\section{Study Site}

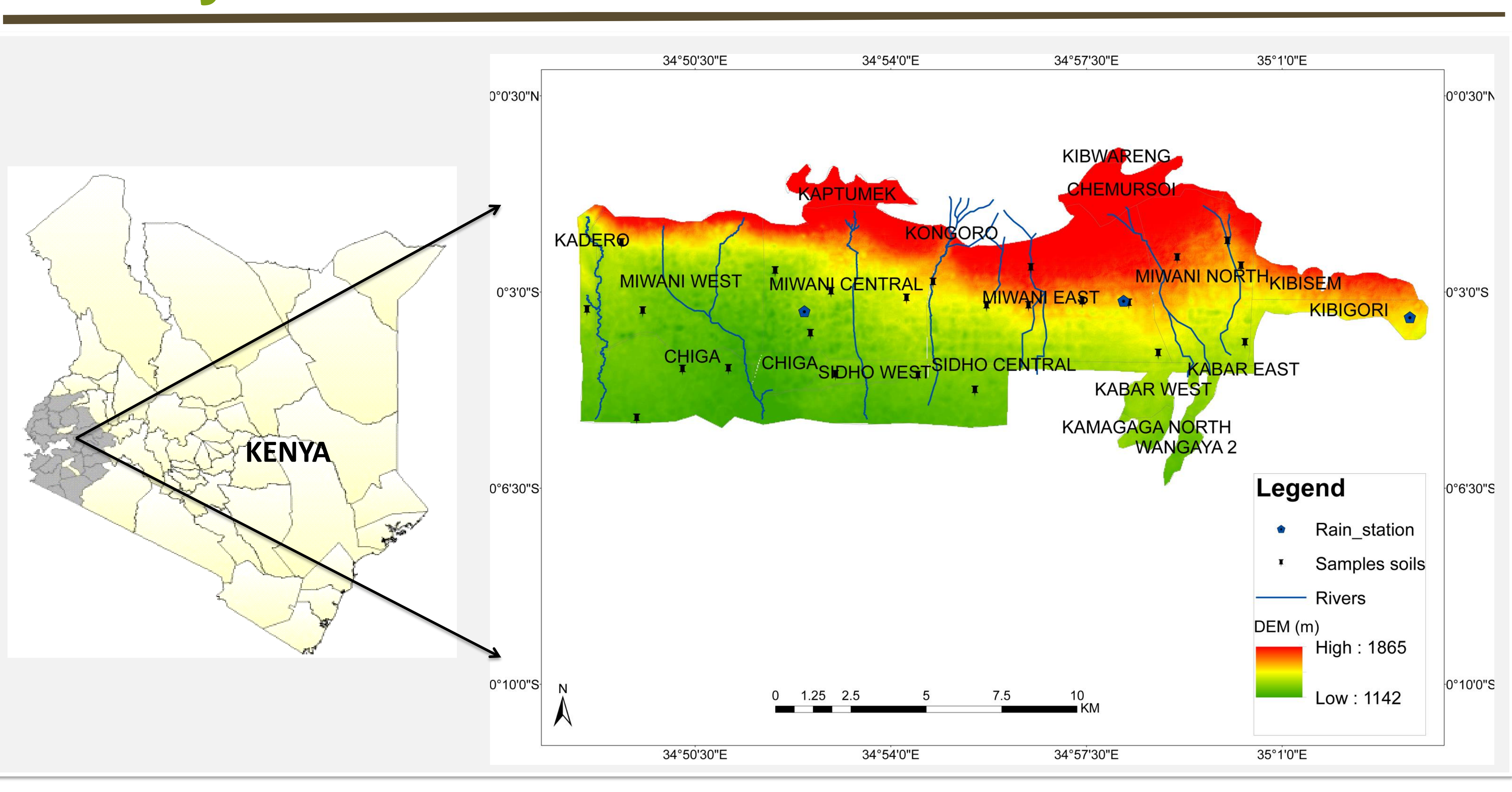

Figure 1: Map of Kibos-Miwani sugarcane zone of Kenya.

Kibos-Miwani sugar zone is located between $34.80^{\circ} \mathrm{E}$ to $35.08^{\circ} \mathrm{E}$ and $0.00^{\circ} \mathrm{S}$ to $0.11^{\circ} \mathrm{S}$, within sub humid agro-ecological zone (Figure 1). The altitude stretches from $1100 \mathrm{~m}$ to $1800 \mathrm{~m}$ in the escarpment. The slope rises from $2 \%$ in the plains to $>20 \%$ in the hilly areas. The area is characterized with rainfall between $1400 \mathrm{~mm}$ to $1550 \mathrm{~mm}$, with black cotton cambisols in the plains and loamy sandy soils in the hills. The area has mixed cropping system with sugarcane taking the largest coverage $(76 \%)$ under multiple planting and harvesting system.

\section{Data and Methods}

\section{(a) Data}

- Satellite data:

(i) MODIS MOD13Q1 $250 \mathrm{~m}$ resolution product (2000-2013) was used to characterize the temporal evolution of Kibos-Miwani landscape and to select appropriate simulation periods for the erosion model.

(ii) Landsat5 $30 \mathrm{~m}$ resolution images acquired in 2011 were used to document the spatial variability of the vegetation conditions in the simulations.

(iii) A 30 m digital elevation model from ASTER was used to compute the aspect, slope and pedoform (curvature) of the landscape.

Ground survey data:

(i) An 11 year measured rainfall data (2001-2012)

(ii) 24 samples of ground survey data on soil characteristics.

\section{(b) Soil erosion modeling}

A fuzzy based dynamic soil erosion model (FuDSEM; Figure 2) was used to model potential soil erosion based on the selected vegetation seasons through spatial aggregation in a GIS [1,2]. FuDSEM has the advantage of simulating both spatial and temporal information at landscape scale.

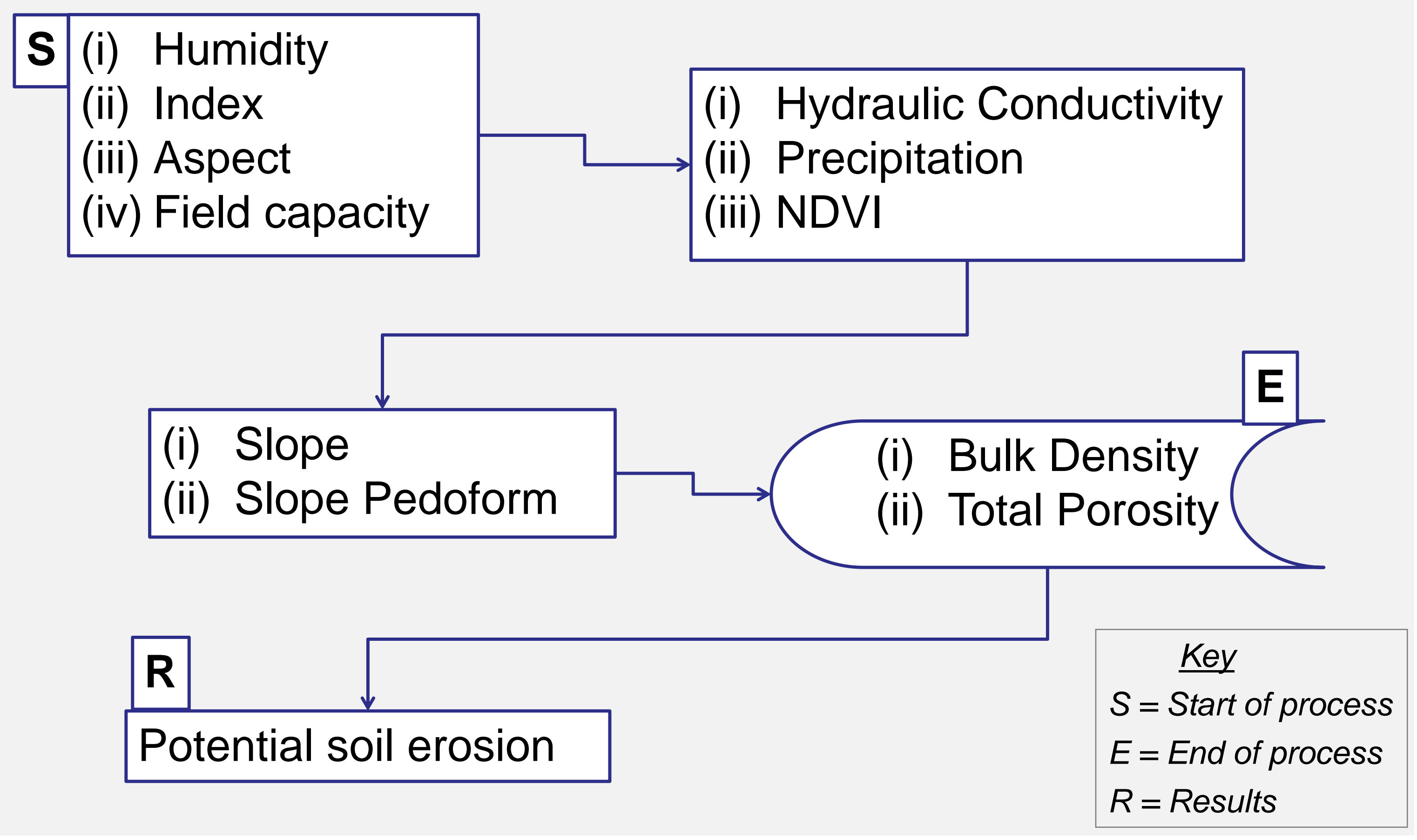

Figure 2. Structure of the fuzzy based soil erosion model (FuDSEM).

\section{Results}

(a) Temporal variability

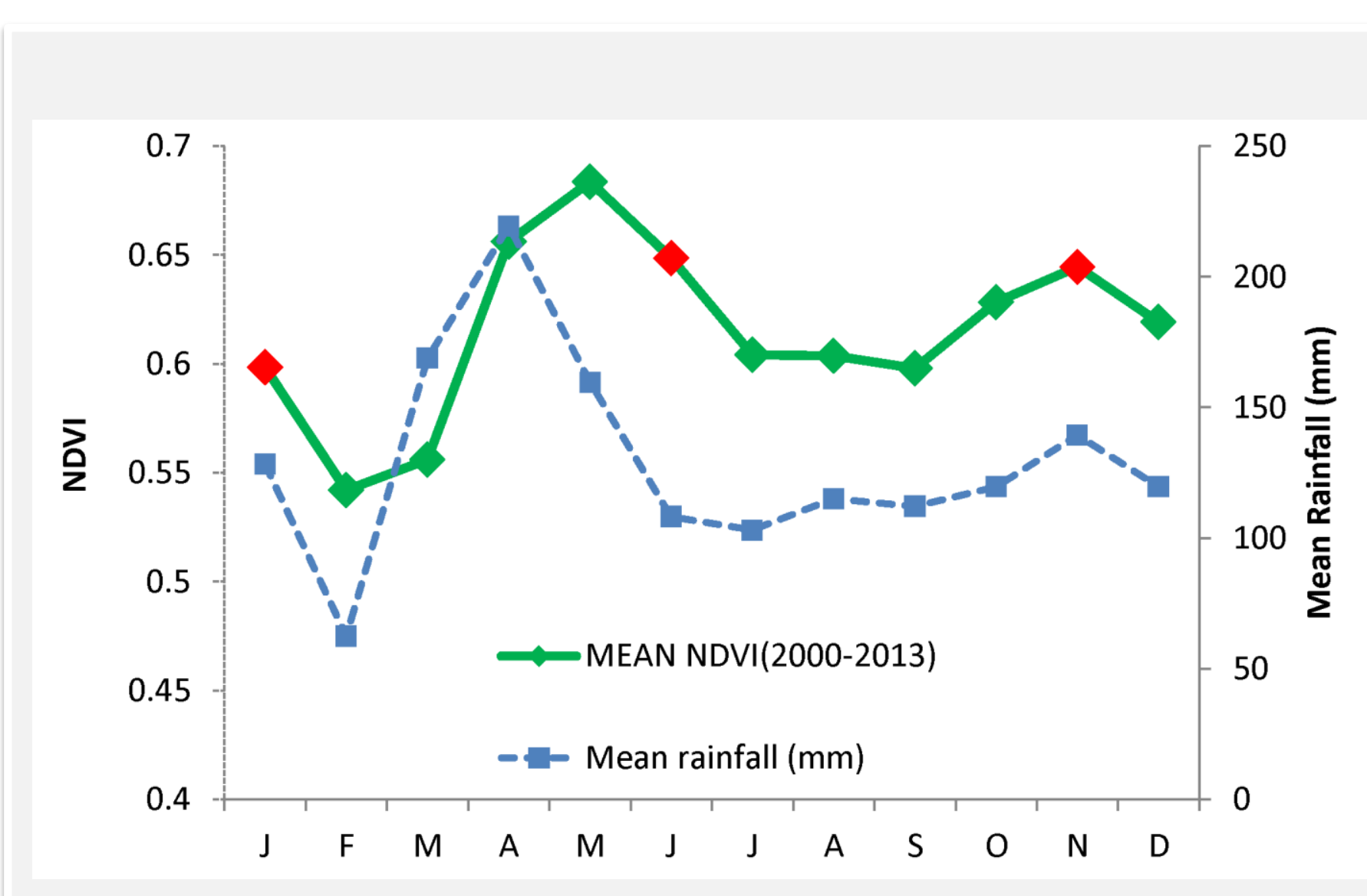

MODIS $250 \mathrm{~m}$ NDVI exhibits two peaks corresponding to the interaction between sugarcane physiology and the bimodal rainfall with a one-month time lag (Figure 3). To calculate erosion risk for the three periods, we selected Landsat5 images corresponding to January and June, that are close to the minimum and maximum (long rain season) vegetation periods respectively; and November which is second maximum vegetative season (short rain season).

Figure 3: Mean seasonal vegetation conditions variability, as measured We infer that rainfall distribution is the main driver of these variations.

by MODIS for the period 2000-2013. In red,

are months selected for FuDSEM simulations.

\section{(b) Spatial variability}

Landsat5 images demonstrated spatial variability in vegetation conditions with vegetated, harvested and planted fields being identified on the image. We infer that crop management is the main driver of these local variations.

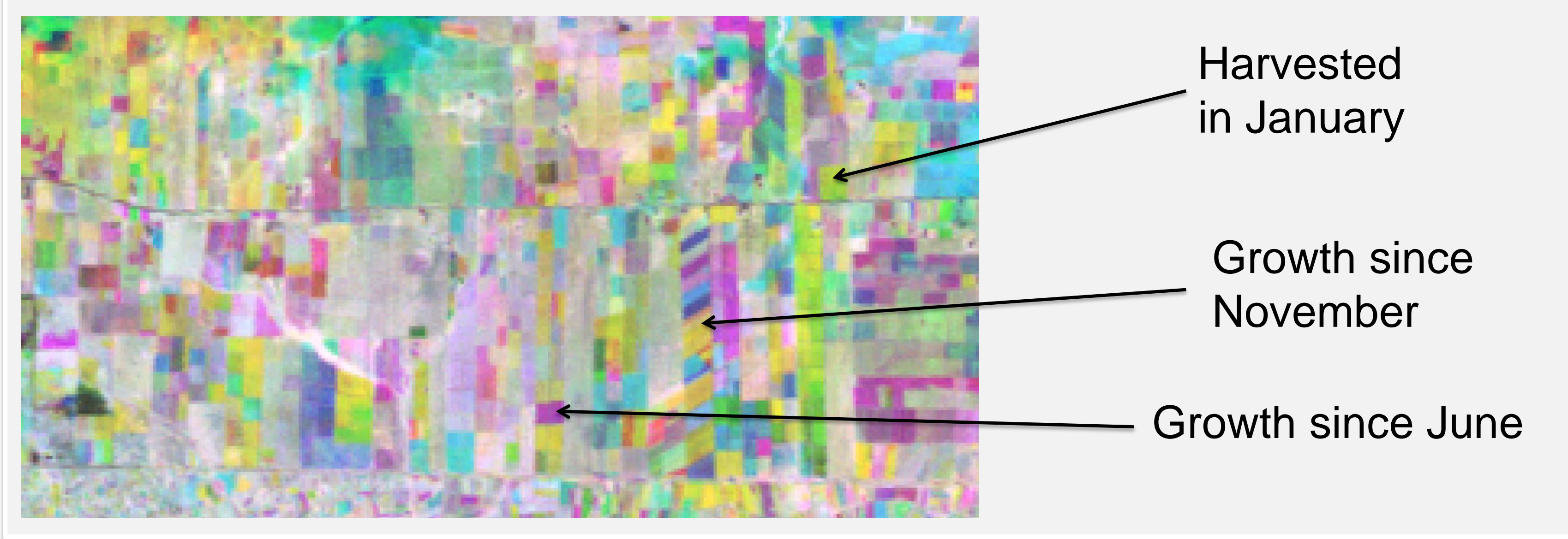

Figure 4: 2011 Landsat NDVI coloured composite image (R: January; G: June; B: November).

\section{(c) Soil erosion simulations}

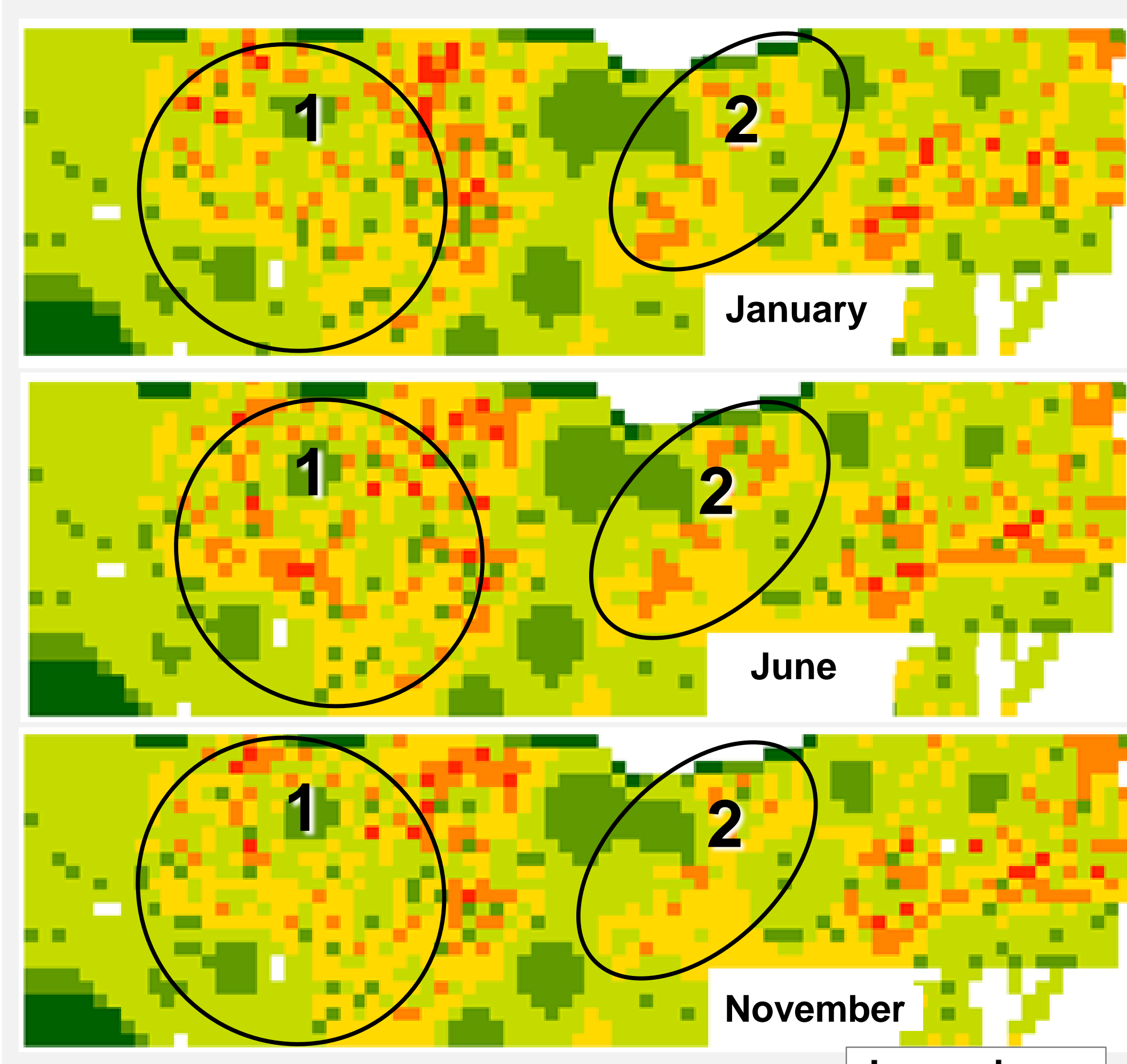

Figure 5: Kibos-Miwan simulated soil erosion maps using the FuDSEM model.
Resultant erosion risk values range from 0.10 in green (lowest) to 0.75 in red (highest). There is homogeneous low erosion in areas with natural land cover, and a mosaic of medium to high erosion in the cropped area. Globally the mean value for erosion (0.42) does not change through time, but November is the period of less erosion in the cropped area (Circle 1 and 2). We therefore infer that enhanced vegetation during the short rain season minimizes erosion.

Additionally, observations on the $30 \mathrm{~m}$ pixels show moderate to intense erosion risk in cropped area (circles 1 and 2). There is variable erosion risk from one pixel to the other in the three simulated months indicating that variability in vegetation conditions at the field scale (Figure 5) influence soil conditions which eventually impact the erosion risk. We infer that crop management practices (planting and harvesting processes) realized in Figure 4 are the drivers of erosion

\section{Conclusion and perspectives}

Satellite imagery has been able to characterize the spatial and temporal dynamics of Kibos-Miwani landscape by identifying relevant images to feed in the erosion model. Results have shown a moderate slope sensitivity to erosion risk and this is alluded to continuous sugarcane cover in the landscape throughout the year.

The next step will be to acquire a complete Landsat8 time series and collect ground data to improve erosion simulations by incorporating information on crop management practices (crop species and sugarcane harvesting process) at field scale derived from image analysis. 